\title{
EMBEDDINGS OF COMPACTA WITH SHAPE DIMENSION IN THE TRIVIAL RANGE
}

\author{
GERARD A. VENEMA
}

\begin{abstract}
In this paper a loop condition is defined which generalizes the cellularity criterion and applies to compacta with nontrivial shape. It is shown that if $X, Y \subset E^{n}, n \geqslant 5$, are compacta which satisfy this loop condition and whose shape classes include a space having dimension in the trivial range with respect to $n$, then $\operatorname{Sh}(X)=\operatorname{Sh}(Y)$ is equivalent to $E^{n}-X$ $\approx E^{n}-Y$. An application is given to compacta with the shape of a compact connected abelian topological group.
\end{abstract}

1. Introduction and statements of main results. Recently several individuals have studied special cases of the following general problem: if $X$ and $Y$ are compacta in Euclidean $n$-space $E^{n}$, under what conditions is $E^{n}-X$ $\approx E^{n}-Y$ equivalent to $\operatorname{Sh}(X)=\operatorname{Sh}(Y)$ ? The results of this paper concern compacta whose shape classes include a space having dimension in the trivial range with respect to $n$. We give a global homotopy condition under which the equivalence holds for such compacta. Before stating our main result we make some definitions.

Definition. Let $X$ be a compact subset of the manifold $M . X$ is said to satisfy the inessential loops condition (ILC) if for every neighborhood $U$ of $X$ in $M$ there exists a neighborhood $V$ of $X$ in $U$ such that each loop in $V-X$ which is null-homotopic in $V$ is also null-homotopic in $U-X$. (See $\S 2$ for the definitions of other loop conditions and a discussion of some of the relationships among them.) For any compactum $X$, the shape dimension of $X(\operatorname{Sd}(X))$ is defined by $\operatorname{Sd}(X)=\min \{\operatorname{dim} Y: \operatorname{Sh}(X)=\operatorname{Sh}(Y)\}$. We say that $k$ is in the trivial range with respect to $n$ if $2 k+2 \leqslant n$.

THEOREM 1. Let $X$ and $Y$ be compacta in $E^{n}, n \geqslant 5$, satisfying ILC and having shape dimension in the trivial range with respect to $n$. Then $E^{n}-X$ $\approx E^{n}-Y$ if any only if $\operatorname{Sh}(X)=\operatorname{Sh}(Y)$.

As a consequence of Theorem 1 we prove the following theorem about compacta with the shape of a topological group. For example, $A$ and $B$ in Theorem 2 could be solenoids. Recall that every finite dimensional compact connected abelian topological group is metrizable [16].

Received by the editors September 22, 1975.

AMS (MOS) subject classifications (1970). Primary 55D99, 57A15, 57A35; Secondary 22B99, 57C30, 57A60.

Key words and phrases. Shape, loop conditions, weak flatness.

${ }^{1}$ The material in this paper will be included in the author's Ph.D. dissertation written under the direction of Professor T. B. Rushing at the University of Utah. Research partially supported by a University of Utah Graduate Research Fellowship. 
TheOrem 2. Let $X, Y \subset E^{n}, n \geqslant 5$, be globally 1-alg compacta and let $A, B$ be compact connected abelian topological groups with $2 \operatorname{dim} A+2 \leqslant n$. If $\operatorname{Sh}(X)=\operatorname{Sh}(A)$ and $\operatorname{Sh}(Y)=\operatorname{Sh}(B)$ then the following are equivalent:

(i) $E^{n}-X \approx E^{n}-Y$,

(ii) $\operatorname{Sh}(X)=\operatorname{Sh}(Y)$, and

(iii) $A$ and $B$ are topologically isomorphic.

Theorem 1 is related to several other recent results. Chapman [3] proved that if $\operatorname{dim} X, \operatorname{dim} Y \leqslant k$ and $3 k+3 \leqslant n$, then there are copies $X^{\prime}$ and $Y^{\prime}$ of $X$ and $Y$, respectively, in $E^{n}$ so that $\operatorname{Sh}(X)=\operatorname{Sh}(Y)$ if and only if $E^{n}-X^{\prime} \approx E^{n}-Y^{\prime}$. Geoghegan and Summerhill [5] refined Chapman's theorem by reducing the unnecessary condition $3 k+3 \leqslant n$ to the trivial range and by making more explicit which copies of $X$ and $Y$ are acceptable. Specifically, they required that the copies of $X$ and $Y$ be 1-ULC. Hollingsworth and Rushing [7] improved this result by replacing 1-ULC (which is a local condition) with the small loops condition (which is global). The global condition is more desirable for a weak flatness theorem of this type-see [7] for more details.

The work of Hollingsworth and Rushing is generalized in Theorem 1 since the same conclusion is drawn for compacta which themselves do not necessarily have dimension in the trivial range but merely have the shape of such. Coram, Daverman and Duvall [4] have previously proved Theorem 1 in the special case that $\operatorname{dim} X \leqslant n-3$ and $Y$ is a finite polyhedron with dimension in the trivial range.

Theorem 2 answers a question raised by J. Keesling.

The author wishes to express his appreciation to Professor T. B. Rushing for a great deal of help with this research.

2. Definitions and notation. Let $X$ be a compactum in the manifold $M^{n} . X$ is said to satisfy the cellularity criterion (small loops condition) if given a neighborhood $U$ of $X$ there exists a neighborhood $V$ of $X$ in $U$ (and a number $\varepsilon>0$ ) such that any loop in $V-X$ (any $\varepsilon$-loop in $V-X)$ is null-homotopic in $U-X . X$ is said to be globally 1 -alg in $M$ if given a neighborhood $U$ of $X$ there exists a neighborhood $V$ of $X$ in $U$ such that any loop in $V-X$ which is null-homologous in $U-X$ is null-homotopic in $U-X$.

These loop conditions are closely related. For example if $\operatorname{dim} X \leqslant n-2$, then ILC is equivalent to the small loops condition. On the other hand, if $X$ has the shape of a point, then ILC is equivalent to the cellularity criterion. In case $\operatorname{Sd}(X) \leqslant n-3$, Alexander duality shows that the inclusion induced homomorphism $H_{1}(V-X) \rightarrow H_{1}(V)$ is an isomorphism. Hence if $\operatorname{Sd}(X)$ $\leqslant n-3, X$ globally 1 -alg implies $X$ satisfies ILC. Finally, it can easily be seen that if $X$ has the shape of the inverse limit of a sequence of ANR's where each of these ANR's has abelian fundamental group, then $X$ is globally 1-alg whenever $X$ satisfies ILC.

Throughout this paper the symbols $\approx$ and $\simeq$ will have the following meanings: ₹ means "is homeomorphic to," "is isomorphic to," or "is topologically isomorphic to," depending on the context; while $\simeq$ means "is homotopic to." $H_{*}$ denotes reduced singular homology, $H^{*}$ Cech cohomology and $H_{c}^{*}$ Alexander cohomology with compact supports [13] all with integer coefficients. 
All spaces are assumed to be metric.

For definitions of concepts related to shape theory, the reader is referred to [1] and [10]. For all other definitions consult [12].

3. Compacta in standard position. We begin by making a definition [3] which is basic to the entire proof of Theorem 1.

Definition. Let $X \subset E^{n}$ be a compactum and $k=\operatorname{Sd}(X) . X$ is in standard position if there exist sequences $\left\{P_{i}\right\}_{i=1}^{\infty}$ and $\left\{N_{i}\right\}_{i=1}^{\infty}$ such that

(i) each $P_{i}$ is a compact polyhedron in $E^{n}, \operatorname{dim} P_{i} \leqslant k$,

(ii) each $N_{i}$ is a regular neighborhood of $P_{i}$ in $E^{n}$,

(iii) each $N_{i+1} \subset$ int $N_{i}$, and

(iv) $X=\bigcap_{i=1}^{\infty} N_{i}$.

If $\operatorname{dim} X=k$ and $2 k+1 \leqslant n$, then the set of embeddings $f: X \rightarrow E^{n}$ such that $f(X)$ is in standard position is a dense $G_{\delta}$-subset of the set of maps of $X$ into $E^{n}\left[5\right.$, Theorem 3.3]. In this section we show that compacta in $E^{n}$ which satisfy ILC and have shape dimension less than $n-2$ are in standard position.

For any pair $(A, B)$, the notation $\pi_{i}(A, B)=0$ means that every map $f$ : $\left(\Delta^{i} \times 0, \partial \Delta^{i} \times 0\right) \rightarrow(A, B)$ extends to a map $\bar{f}:\left(\Delta^{i} \times[0,1], \partial \Delta^{i} \times[0,1] \cup\right.$ $\left.\Delta^{i} \times 1\right) \rightarrow(A, B) .\left(\Delta^{i}\right.$ denotes the standard $i$-simplex.)

LEMma 1. Let $X \subset E^{n}$ be a compactum satisfying ILC and let $k=\operatorname{Sd}(X)$. Then $\pi_{i}(U, U-X)=0,0 \leqslant i \leqslant n-k-1$, for every compact neighborhood $U$ of $X$ in $E^{n}$.

Proof. It may be assumed that $U$ is connected because otherwise the following proof can be applied to each component of $U$. Since $\operatorname{Sd}(X)=k$, there exists a compactum $Y$ with $\operatorname{dim} Y=k$ and $\operatorname{Sh}(X)=\operatorname{Sh}(Y)$. Embed $Y$ in $E^{2 k+1}$ in standard position; say $Y=\bigcap_{i=1}^{\infty} N_{i}$ where each $N_{i}$ is a regular neighborhood of $P_{i}$ in $E^{2 k+1}, \operatorname{dim} P_{i} \leqslant k$ and $N_{i+1} \subset$ int $N_{i}$.

We first construct a convenient sequence of neighborhoods whose intersection is $X$. Let $\left\{f_{i}, X, Y\right\}_{E^{n}, E^{2 k+1}}$ and $\left\{g_{i}, Y, X\right\}_{E^{2 k+1}, E^{n}}$ be fundamental sequences which show that $X$ and $Y$ have the same shape [2, Theorem 2.4]. Choose an integer $j$ such that $g_{i}\left(N_{j}\right) \subset U$ for almost all $i$. Now choose a neighborhood $V$ of $X$ such that $f_{i}(V) \subset N_{j}$ and $g_{i} f_{i} \mid V \simeq 1_{V}$ in $U$ for almost all $i$. It may be assumed that $g_{i} \mid P_{j}$ is piecewise linear. Thus the inclusion map $\beta: V \hookrightarrow U$ is homotopic in $U$ to a map of $V$ into $g_{i}\left(P_{j}\right)$. Inductively then we can construct a sequence of neighborhoods $V_{i}$ in $U$ and polyhedra $K_{i}$ such that $\operatorname{dim} K_{i} \leqslant k, V_{i} \cup K_{i} \subset V_{i-1}, \cap_{i=1}^{\infty} V_{i}=X$ and the inclusion map $V_{i}$ $\rightarrow V_{i-1}$ is homotopic in $V_{i-1}$ to a map of $V_{i}$ into $K_{i}$.

Consider the universal cover $p: \tilde{U} \rightarrow U$. Denote $p^{-1}\left(V_{i}\right)$ by $\bar{V}_{i}$ and $p^{-1}(X)$ by $\bar{X}$. We show that $H_{c}^{q}(\bar{X})=0$ for $q>k$. Let $f: V_{i} \times[0,1] \rightarrow V_{i-1}$ be a homotopy such that $f_{0}=1_{V_{i}}$ and $f_{1}\left(V_{i}\right) \subset K_{i}$. The diagram

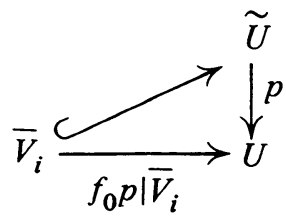


commutes, so $f_{t} p \mid \bar{V}_{i}$ can be lifted to a homotopy $g_{t}$. Since $g_{1}\left(\bar{V}_{i}\right) \subset p^{-1}\left(K_{i}\right)$ and $p$ is a local homeomorphism, $\operatorname{dim} g_{1}\left(\bar{V}_{i}\right) \leqslant k$.

Let $\bar{V}_{i}^{+}$and $\bar{X}^{+}$denote the one-point compactifications of $\bar{V}_{i}$ and $\bar{X}$ respectively. Since $g_{t}$ is a proper map, $g_{t}$ can be extended to $\bar{g}_{t}: \bar{V}_{i}^{+} \rightarrow \bar{V}_{i-1}^{+}$. Hence the inclusion $\bar{V}_{i}^{+} \hookrightarrow \bar{V}_{i-1}^{+}$factors up to homotopy through a map into a space of dimension at most $k$. Thus the continuity axiom implies that $H^{q}\left(\bar{X}^{+}\right)=0$ for $q>k$. Finally [13, Corollary 6.6.12] shows that $H_{c}^{q}(\bar{X})$ $=0, q>k$.

Now Alexander duality [13, Theorem 6.9.10] gives $H_{q}(\tilde{U}, \tilde{U}-\bar{X})$ $\approx H_{c}^{n-q}(\bar{X}) \approx 0$ for $n-q \geqslant k+1$. We look at the homology sequence of the pair $(\tilde{U}, \tilde{U}-\bar{X})$ and see that $H_{0}(\tilde{U}-\bar{X})=0$. So $\tilde{U}-\bar{X}$ is connected.

$\pi_{1}(U, U-X)=0$ since if $f:\left(\Delta^{1} \times 0, \partial \Delta^{1} \times 0\right) \rightarrow(U, U-X), f$ can be lifted to $f^{\prime}:\left(\Delta^{1} \times 0, \partial \Delta^{1} \times 0\right) \rightarrow(\tilde{U}, \tilde{U}-\bar{X}) . \tilde{U}-\bar{X}$ is connected, so $f^{\prime}(0)$ and $f^{\prime}(1)$ can be joined by an arc in $\tilde{U}-\bar{X}$. The resulting loop is null-homotopic in $U$. The projection down of this homotopy is the desired homotopy in $U$.

We next prove that $\pi_{1}(\tilde{U}-\bar{X})=0$. Let $f: S^{1} \rightarrow \tilde{U}-\bar{X}$ be a loop. $f$ extends to $\bar{f}: \Delta^{2} \rightarrow \tilde{U}$. Consider $p \bar{f}:\left(\Delta^{2}, S^{1}\right) \rightarrow(U, U-X)$. Choose $V \subset U$ to be a neighborhood of $X$ satisfying the inessential loops condition relative to $U$. Now triangulate $\Delta^{2}$ so that each simplex whose image intersects $X$ is mapped into $V$. Using the fact that $\pi_{1}(V, V-X)=0$ (by the same argument as was used to show that $\left.\pi_{1}(U, U-X)=0\right)$, we can push the image of the 1 -skeleton of this triangulation off $X$. If $\sigma$ is a 2-simplex in $\Delta^{2}, p \bar{f} \mid \partial \sigma$ : $\partial \sigma \rightarrow V-X$ and $p \bar{f} \mid \partial \sigma \simeq 0$ in $V$, so $p \bar{f} \mid \sigma$ may be replaced by a map of $\sigma$ into $U-X$ which agrees with $p \bar{f}$ on $\partial \sigma$. Thus $p \bar{f} \mid S^{1} \simeq 0$ in $U-X$. Lifting this homotopy, we see that $f \simeq 0$ in $\tilde{U}-\bar{X}$.

Finally, we apply the relative Hurewicz Theorem [13, Theorem 7.5.4] and conclude that $\pi_{i}(\tilde{U}, \tilde{U}-\bar{X}) \approx H_{i}(\tilde{U}, \tilde{U}-\bar{X})=0,2 \leqslant i \leqslant n-k-1$. The homotopy lifting property now can be used to show that $\pi_{i}(U, U-X)=0$, $i \leqslant n-k-1$.

LeMMA 2. Let- $X \subset E^{n}$ be a compactum such that $\operatorname{Sd}(X) \leqslant n-3$. Given a neighborhood $U$ of $X$ there exists a neighborhood $V$ of $X$ such that for any compact polyhedron $K \subset V$ with $\operatorname{dim} K \leqslant n-3$ there is a polyhedron $P$ and $a$ regular neighborhood $N$ of $P$ such that $K \subset$ int $N \subset N \subset U$ and $\operatorname{dim} P$ $\leqslant \operatorname{Sd}(X)$.

Proof. The proof is by induction on $k=\operatorname{dim} K$. If $k \leqslant \operatorname{Sd}(X), P=K$ and $V=U$ will work; so it may be assumed that $k>\operatorname{Sd}(X)$ and that the lemma is true for polyhedra of dimension less than $k$. Choose $V^{\prime} \subset U$ using this inductive hypothesis. As before choose a neighborhood $V$ of $X$ in $V^{\prime}$ and a polyhedron $P^{\prime} \subset V^{\prime}$ such that $\operatorname{dim} P^{\prime} \leqslant \operatorname{Sd}(X)$ and the inclusion $A \hookrightarrow V^{\prime}$ is homotopic in $V^{\prime}$ to a map of $V$ into $P^{\prime}$.

Let $f: K \times[0,1] \rightarrow V^{\prime}$ be a homotopy such that $f_{0}=1_{K}$ and $f_{1}(K) \subset P^{\prime}$. By Zeeman's Piping Lemma [15, Lemma 48], we may assume that there exists a polyhedron $J \subset K \times I$ such that

(1) $S(f) \subset J$,

(2) $\operatorname{dim} J \leqslant 2 k-n+2 \leqslant k-1$,

(3) $\operatorname{dim}\left[J \cap\left(K^{k-1} \times[0,1]\right)\right] \leqslant 2 k-n+1 \leqslant k-2$, and

(4) $K \times[0,1] \rightarrow J \cup\left(K^{k-1} \times[0,1]\right) \cup K \times 1$.(Here $K^{i}$ denotes the $i$ skeleton of $K$.) 
Let $L$ be a $(k-2)$-dimensional subpolyhedron of $K$ such that $L \supset K^{k-2}$ and $L \times[0,1] \supset J \cap\left(K^{k-1} \times[0,1]\right)$. By induction it may be assumed that $f(L \times[0,1] \cup J) \cup P^{\prime} \subset N \subset U$ where $N$ is a regular neighborhood of some polyhedron $P$ with dimension $\leqslant \operatorname{Sd}(X)$. It remains only to engulf $(k-1)$ - and $k$-simplexes of $K$.

$$
K^{k-1} \times[0,1] \searrow K^{k-2} \times[0,1] \cup K^{k-1} \times 1 \cup\left(L \cap K^{k-1}\right) \times[0,1] .
$$

The image of the latter set is already contained in $N$ and contains $S\left(f \mid K^{k-1} \times[0,1]\right)$. Following the image of this collapse, $K^{k-1}$ can be engulfed with $N$. Similarly $K \times[0,1] \searrow J \cup K^{k-1} \times[0,1] \cup K \times 1$, so $N$ can be pushed out to cover all of $K$.

Lemma 3. Let $X \subset E^{n}, n \geqslant 5$, be a compactum with $\operatorname{Sd}(X) \leqslant n-3$. Then $X$ satisfies ILC if and only if $X$ is in standard position.

Proof. It suffices to show that given a neighborhood $U$ of $X$ there exists a polyhedron $P$ in $U$ with $\operatorname{dim} P \leqslant \operatorname{Sd}(X)$ and a regular neighborhood $N$ of $P$ such that $X \subset$ int $N \subset N \subset U$. Let $V \subset U$ be given by Lemma 2 and let $M$ be a compact PL manifold neighborhood of $X$ in $V$. Denote the $(n-3)$ skeleton of $M$ by $M^{n-3}$ and the dual 2-skeleton by $M_{*}^{2}$. By Lemma 2 there exists a polyhedron $P$ with $\operatorname{dim} P \leqslant \operatorname{Sd}(X)$ and a regular neighborhood $N$ of $P$ such that $M^{n-3} \subset$ int $N \subset N \subset U$.

By Lemma 1 and Stallings' engulfing theorem [14], there exists a PL homeomorphism $h_{1}$ : int $M \rightarrow$ int $M$ with compact support such that $h_{1}$ (int $\left.M-X\right) \supset M_{*}^{2} \cap$ int $M$. Extend $h_{1}$ via the identity to $U$. Let $h_{2}$ be a homeomorphism of $U$ which pushes $N$ across the join structure between $M^{n-3}$ and $M_{*}^{2}$ until $M \subset h_{1}(U-X) \cup h_{2}$ (int $\left.N\right)$. Then $h_{1}^{-1} h_{2}(N)$ is the regular neighborhood we are looking for.

4. Proofs of Theorems 1 and 2. In this section we complete the proof of Theorem 1 and prove Theorem 2. The following lemma is used to keep an inductive argument going in the proof of Theorem 1.

Lemma 4. Let $X, Y \subset E^{n}, n \geqslant 5$, be compacta in standard position with shape dimensions in the trivial range with respect to $n$ and let $\left\{f_{i}, X, Y\right\}$ and $\left\{f_{i}^{\prime}, Y, X\right\}$ be fundamental sequences which are homotopy inverse to one another. Let $U_{0}$ be a neighborhood of $X$ and $h$ be a PL homeomorphism of $E^{n}$ such that $Y \subset h\left(U_{0}\right)$ and such that there exists a neighborhood $W_{0}$ of $Y$ with $h^{-1}\left|W_{0} \simeq f_{i}^{\prime}\right| W_{0}$ in $U_{0}$ for almost all $i$. Then for every open set $V_{0}, Y \subset V_{0}$ $\subset h\left(U_{0}\right)$, there exists a PL homeomorphism $q$ of $E^{n}$ such that $q \mid E^{n}-U_{0}$ $=h \mid E^{n}-U_{0}, X \subset q^{-1}\left(V_{0}\right)$, and $q\left|U_{1} \simeq f_{i}\right| U_{1}$ in $V_{0}$ for almost all $i$ where $U_{1}$ is some neighborhood of $X$.

Proof. Assume that $X=\bigcap_{j=1}^{\infty} M_{j}$ where each $M_{j}$ is a regular neighborhood of a compact polyhedron $L_{j}$ and that $\operatorname{dim} L_{j} \leqslant \operatorname{Sd}(X)$. Choose neighborhoods $V \subset V_{0}$ of $Y$ and $U \subset U_{0}$ of $X$ and an integer $i_{0}$ such that $h^{-1}\left|V \simeq f_{i}^{\prime}\right| V$ in $U_{0}, f_{i}\left|U \simeq f_{i+1}\right| U$ in $V$, and $f_{i}^{\prime} f_{i} \mid U \simeq 1_{U}$ in $U_{0}$ for $i \geqslant i_{0}$. Let $j$ be an integer large enough so that $M_{j} \subset U . f_{i_{0}} \mid L_{j}$ can be approximated by a PL embedding $\hat{f}$.

Notice that $h^{-1} \circ \hat{f} \simeq f_{i_{0}}^{\prime} \circ \hat{f} \simeq f_{i_{0}}^{\prime} \circ f_{i_{0}} \mid L_{j} \simeq 1_{L_{j}}$ in $U_{0}$. So $\hat{f} \simeq h \mid L_{j}$ in $h\left(U_{0}\right)$. Hence there exists a PL homeomorphism $r$ of $E^{n}$ which is the identity outside $h\left(U_{0}\right)$ and such that $r h \mid L_{j}=\hat{f}[6]$. It may be assumed 
that $r h\left(M_{j}\right) \subset V$. Taking $q=r h$ and $U_{1}=M_{j}$ gives the desired conclusion.

Proof of Theorem 1. Suppose that $\operatorname{Sh}(X)=\operatorname{Sh}(Y)$. A homeomorphism of $E^{n}-X$ onto $E^{n}-Y$ can be constructed using the technique of [5, Lemma 4.2]. Lemma 4 above replaces Lemma 4.1 of [5]. Now suppose that $E^{n}-X$ $\approx E^{n}-Y$. There exist $X^{\prime}$ and $Y^{\prime}$ with dimension in the trivial range satisfying $\operatorname{Sh}(X)=\operatorname{Sh}\left(X^{\prime}\right)$ and $\operatorname{Sh}(Y)=\operatorname{Sh}\left(Y^{\prime}\right)$. It may be assumed that $X^{\prime}$ and $Y^{\prime}$ are embedded in $E^{n}$ as 1-ULC subsets [7, Lemmas 2 or 5, §3]. The first part of the theorem implies that $E^{n}-X^{\prime} \approx E^{n}-Y^{\prime}, \operatorname{so} \operatorname{Sh}(X)=\operatorname{Sh}(Y)$ by [5] again.

Proof of Theorem 2. The implication (iii) $\Rightarrow$ (ii) is obvious and (ii) $\Rightarrow$ (iii) is exactly Corollary 1.3 of [9]. Theorem 1 gives (iii) $\Rightarrow$ (i). Our proof that (i) $\Rightarrow$ (iii) actually establishes a stronger result which we state as Theorem 3.

Theorem 3. Let $X, Y \subset E^{n}$ be compacta and let $A, B$ be compact connected abelian topological groups with $\operatorname{Sh}(X)=\operatorname{Sh}(A)$ and $\operatorname{Sh}(Y)=\operatorname{Sh}(B)$. Then $E^{n}-X \approx E^{n}-Y$ implies $A \approx B$.

Proof. Suppose $E^{n}-X \approx E^{n}-Y$. Then by Alexander duality [13, Theorem 6.2.16] $H^{1}(X) \approx H^{1}(Y)$; hence $H^{1}(A) \approx H^{1}(B)$ [10, Theorem 16]. Therefore $\operatorname{char} A \approx \operatorname{char} B$ [8, Theorem 1.4] and so Pontryagin duality [11, Theorem 52] shows that $A \approx B$.

\section{REFERENCES}

1. K. Borsuk, Concerning homotopy properties of compacta, Fund. Math. 62 (1968), 223-254. MR $37 \# 4811$.

2. , Remark on a theorem of S. Mardešic, Bull. Acad. Polon. Sci. Sér. Sci. Math. Astronom. Phys. 19 (1971), 475-483. MR 48 \#2986.

3. T. A. Chapman, Shapes of finite dimensional compacta, Fund. Math. 76 (1972), no. 3, 261-276. MR 47 \#9531.

4. D. Coram, R. Daverman and P. Duvall, Jr., A loop condition for embedded compacta, Proc. Amer. Math. Soc. 53 (1975), 205-212.

5. R. Geoghegan and R. R. Summerhill, Concerning the shapes of finite dimensional compacta, Trans. Amer. Math. Soc. 179 (1973), 281-292. \#3456.

6. H. Gluck, Embeddings in the trivial range, Ann. of Math. (2) 81 (1965), 195-210. MR 30

7. J. G. Hollingsworth and T. B. Rushing, Embeddings of shape classes of compacta in the trivial range (to appear).

8. J. Keesling, An algebraic property of the $\check{C}$ ech cohomology groups which prevents local connectivity and movability, Trans. Amer. Math. Soc. 190 (1974), 151-162.

9. , Shape theory and compact connected abelian topological groups, Trans. Amer. Math. Soc. 194 (1974), 349-358. MR 49 \#9803.

10. S. Mardešic and J. Segal, Shapes of compacta and ANR-systems, Fund. Math. 72 (1971), no. 1, 41-59. MR 45 \#686.

11. L. S. Pontrjagin, Continuous groups, 2nd ed., GITTL, Moscow, 1954; English transl., Topological groups, Gordon and Breach, New York, 1966. MR 17, 171; 34 \# 1439.

12. T. B. Rushing, Topological embeddings, Pure and Appl. Math., vol. 52, Academic Press, New York and London, 1973. MR 50 \# 1247.

13. E. H. Spanier, Algebraic topology, McGraw-Hill, New York, 1966. MR 35 \# 1007.

14. J. R. Stallings, The piecewise-linear structure of Euclidean space, Proc. Cambridge Philos. Soc. 58 (1962), 481-488. MR 26 \#6945.

15. E. C. Zeeman, Seminar on combinatorial topology, Mimeographed notes, Inst. Hautes Études Sci., Paris, 1963.

16. J. D. Newburgh, Metrization of finite dimensional groups, Duke Math. J. 20 (1953), 287-293. MR 14, 949. 